also evaluated the relationship between culture positivity, IA, and clinical outcomes.

Methods: A retrospective cohort of THA/TKA PJIs, from 2009 to 2016, were identified by ICD codes, and confirmed by chart review. IA diagnosis was also confirmed by use of IA-specific medications. CN cases were defined as PJIs with no evidence of microbial growth in intraoperative cultures and CP PJI cases were defined by positive microbial growth in intraoperative cultures. Treatment failure was defined as subsequent surgical treatment for infection after the initial infection surgery. H\&E slides of OA and IA PJI cases matched by age (+/-5) sex, and culture status were reviewed by a pathologist for evidence of the histopathologic features listed in Table 2. Fisher's exact test, chi-square test, and Kaplan-Meier estimates were used.

Results: 807 PJI cases were identified including 36 IA (33 RA and 3 SLE) and 771 OA. A higher proportion of IA PJI were CN $(N=10,27 \%)$ vs. OA PJI ( $N=109$, $14 \%, p=0.02)$. IA-PJI were younger, female, on glucocorticoids, and with more comorbidities. Type of surgical treatment did not differ significantly between IA and OA groups. Comparing CN-IA vs. CP-IA, no difference was observed in age, smoking, diabetes, surgical treatment, IA-specific meds or Charlson comorbidities. One-year survivorship of $\mathrm{CN}-\mathrm{IA}$ and $\mathrm{CN}-\mathrm{OA}$ were $66 \%$ and $87 \%$ ( $p>0.05)$. Across all CP cases, $57 \%$ were staphylococcal, with no differences between groups. Treatment failure was more frequent for CP-IA (42\%) compared to CP-OA $(30 \%),(p=0.2)$

Histopathology of 88 PJIs (31 IA and 57 OA) was reviewed. The IA cohort presented with more chronic inflammation $(p=0.001)$ than the OA cohort. Within the IA cohort, a higher proportion of CP-IA had $>10 P M N$ per HPF $(p=0.003)$ and met MSIS criteria $(p=0.009)$. Comparing CP-OA and $\mathrm{CN}-\mathrm{OA}$, there were no significant differences in histopathology findings or number of patients meeting MSIS criteria.

Conclusion: IA PJls are more likely to be culture negative than OA PJIs. Although our analysis was limited by our cohort size, our findings including differences in histopathology, and better clinical outcomes suggest the presence of biologic differences between $\mathrm{CN}$ and $\mathrm{CP} P \mathrm{PI}$ that require further study.

TABLE 1. Patient characteristics in IA and OA PJIs

\begin{tabular}{|c|c|c|c|c|c|}
\hline & \multicolumn{2}{|c|}{ IA } & \multicolumn{2}{|c|}{ OA } & \multirow[b]{2}{*}{$\mathrm{p}$-value } \\
\hline & $\mathbf{N}$ & $\% / S D$ & $\mathrm{~N}$ & $\% / S D$ & \\
\hline Total & 36 & & 771 & & \\
\hline Age & 58.5 & 11.4 & 66.8 & 12 & $<.001$ \\
\hline BMI & 30.2 & 6.7 & 30 & 6.7 & 0.861 \\
\hline Female & 28 & 77.8 & 332 & 43.1 & $<.001$ \\
\hline $\mathrm{CCl}$ & 2.8 & 1.7 & 1.7 & 2.1 & 0.002 \\
\hline Smoking & 4 & 11.1 & 86 & 11.2 & 0.792 \\
\hline Glucorticoids & 10 & 27.8 & 39 & 5.1 & $<.001$ \\
\hline Culture Negative & 10 & 27.8 & 109 & 14.1 & 0.024 \\
\hline Treatment Success at 2 years & 19 & 52.8 & 509 & 66 & 0.146 \\
\hline
\end{tabular}

IA- inflammatory arthritis; OA - osteoarthritis; PJI -prosthetic joint infection; CCI - Charlson Comorbidity Index

TABLE 2. Histopathology and clinical presentation in IA and OA PJIs

\begin{tabular}{|c|c|c|c|c|c|c|}
\hline & $\mathrm{OA}(\mathrm{N}=57)$ & $\mathrm{IA}(\mathrm{N}=31)$ & \multirow[b]{2}{*}{$\mathrm{p}$-value } & \multirow{2}{*}{$\frac{C P-I A(N=23)}{N(\%)}$} & \multirow[t]{2}{*}{$\mathrm{CN}-\mathrm{IA}(\mathrm{N}=8)$} & \multirow[b]{2}{*}{$\mathrm{p}$-value } \\
\hline & \multicolumn{2}{|l|}{$N(\%)$} & & & & \\
\hline \multicolumn{7}{|l|}{ Pathology Review } \\
\hline >10 PMN per HPF & $42(74)$ & $22(71)$ & 0.806 & $20(87)$ & $2(25)$ & 0.003 \\
\hline Chronic Inflammation & $13(23)$ & $23(74)$ & 0.001 & $18(78)$ & $5(63)$ & 0.393 \\
\hline Necrosis & $17(30)$ & $9(29)$ & 1 & $8(35)$ & $1(13)$ & 0.38 \\
\hline \multicolumn{7}{|l|}{ Clinical Presentation } \\
\hline MSIS & $50(88)$ & $26(84)$ & 0.747 & $22(96)$ & $4(50)$ & 0.009 \\
\hline Sinus Tract & 7 (12) & 7 (23) & 0.233 & $5(22)$ & $2(25)$ & 1 \\
\hline Elevated ESR or CRP & $41(72)$ & $24(77)$ & 0.622 & $17(74)$ & 7 (88) & 1 \\
\hline Elevated Synovial WBC & $33(58)$ & $19(61)$ & 0.823 & $13(57)$ & $6(75)$ & 1 \\
\hline Elevated Synovial \%PMN & $31(54)$ & $20(65)$ & 0.377 & $14(61)$ & $6(75)$ & 0.333 \\
\hline
\end{tabular}

OA - osteoarthritis; IA - inflammatory arthritis; CP - culture positive; CN - culture negative; MSIS - meets Musculoskeletal Infection Society diagnostic criteria

Disclosure of Interests: Milan Kapadia: None declared, Tania Pannellini: None declared, Carine Moezinia: None declared, Andy Miller: None declared, Mark Figgie: None declared, Peter Sculco: None declared, Michael Cross: None declared, Michael Henry: None declared, Linda Russell: None declared, Laura Donlin Consultant of: Consultant - Genentech/Roche, Allina Nocon: None declared, Susan Goodman Shareholder of: Reginosine- Investment, Grant/research support from: Novartis, Horizon, Consultant of: Novartis, Celgene, UCB

DOI: 10.1136/annrheumdis-2020-eular.4777

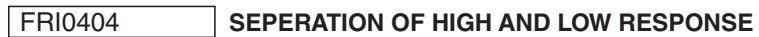 GROUPS IN OSTEOARTHRITIS USING SERUM CARTILAGE DEGRADATION AND FORMATION MARKERS - A 3 YEAR FOLLOW-UP ON THE FORWARD STUDY TESTING THE EFFICACY OF SPRIFERMIN}

H. Gühring ${ }^{1}$, A. C. Bay-Jensen ${ }^{2}$, F. Moreau' ${ }^{1}$, J. R. Andersen ${ }^{3}$, A. R. Bihlet ${ }^{3}$, M. Karsdal ${ }^{2} .{ }^{1}$ Merck KGaA, Darmstadt, Germany; ${ }^{2}$ Nordic Bioscience, Immunoscience, Herlev, Denmark; ${ }^{3}$ Nordic Bioscience, Clinical development, Herlev, Denmark

Background: In osteoarthritis (OA), response to different interventions could be more pronounced in different endotypes of cartilage turnover. Data from UK biobank suggest that an endotype of low cartilage repair is associated with structural progression in osteoarthritis $(\mathrm{OA})[1]$. Sprifermin a truncated and recombinant FGF18 have been shown to induce chondrocyte proliferation and cartilage formation in in vitro settings[2-4]. Efficacy on cartilage thickness in OA was recently demonstrated in the FORWARD study[5].

Objectives: We investigated markers of cartilage formation (serum PRO-C2) and degradation (urine CTX-II), to assess profiles indicative of chondrocyte metabolic activity would be associated with both structural and symptomatic responses to sprifermin.

Methods: Serum and urine from participants of the FORWARD study, a phase II clinical trial testing the efficacy of intra-articular (IA) sprifermin, were collected throughout the study. Clinical data recorded at baseline, year 2 and year 3 follow-up were used, including cartilage thickness and WOMAC. All available baseline samples of the placebo and treatment arms were assessed for serum PRO-C2 and urinary CTX-II. Patients were separated into dichotomized groups based on 33,50 or 66 percentiles cut-points and two-year treatment responses were compared in a prospective-retrospective statistical design manner.

Results: Patients with low baseline PRO-C2 $(<66 \%)$, in the $100 \mathrm{ug} / 4 \mathrm{x}$ treatment arm, had greater difference to placebo in cartilage thickness as compared to the high PRO-C2 and all-comers groups ( 0.06 vs 0.03 and $0.05 \mathrm{~mm}$, respectively) at two and three years (Fig.). Similar effect was seen for low CTX-II (<50\%) $(0.07$ vs -0.03 and $0.05 \mathrm{~mm}$, respectively). In addition, the WOMAC placebo effect was reduced in the low PRO-C2 and the low CTX-II groups, resulting in a difference compared to placebo of -0.81 vs. 1.35 (low vs high proC2 and -2.35 vs 1.47 (low vs high CTX-II).

Conclusion: We noticed that low baseline PRO-C2 and CTX-II indicative for low metabolic activity of chondrocytes were associated with improved symptomatic outcome and slightly increased cartilage thickness compared to high metabolic activity. The parallelism between PRO-C2 and CTX-II point towards the existence of a low cartilage repair endotype and might reflect a subgroup of patients with higher sensitivity towards interventions - an effect that was maintained over three years.

Fig. 1: Longitudinal change in cartilage thickness $(\mathrm{mm})$ in the total femorotibial joint in patients with low and high levels of PRO-C2 (top panel) and CTX-II (lower panel). Data are shown as mean with 95\% $\mathrm{Cl}$ of absolute change from baseline up to 3 Years.
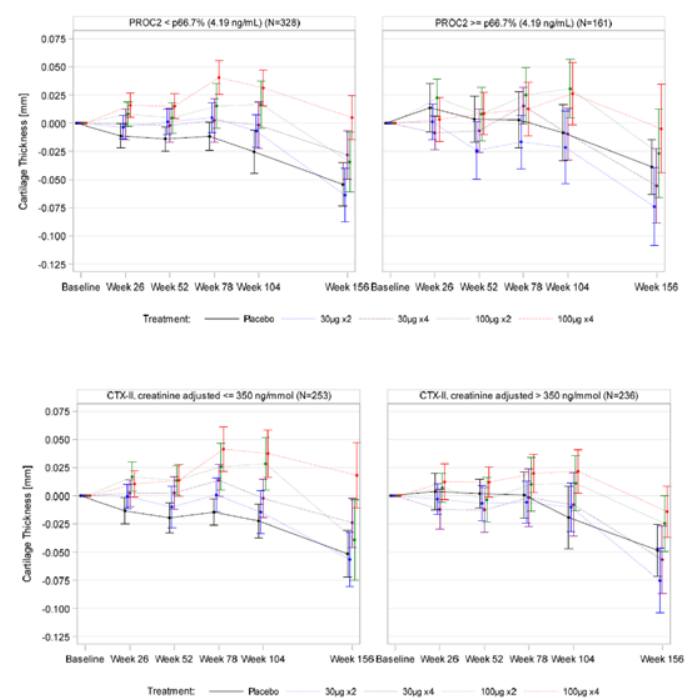
References:

[1] Tachmazidou I, et al. "Identification of new therapeutic targets for osteoarthritis through genome-wide analyses of UK Biobank data." Nat Genet 2019;51:230-6.

[2] Gigout A, et al. "Sprifermin (rhFGF18) enables proliferation of chondrocytes producing a hyaline cartilage matrix". Osteoarthr Cartil. 2017;25.

[3] Reker D et al. "Sprifermin (rhFGF18) modulates extracellular matrix turnover in cartilage explants ex vivo". J Transl Med. 2017;15.

[4] Luo $Y$ et al. "A Novel High Sensitivity Type II Collagen Blood-Based Biomarker, PRO-C2, for Assessment of Cartilage Formation". Int J Mol Sci 2018;19:3485

[5] Hochberg MC et al. "Effect of Intra-Articular Sprifermin vs Placebo on Femorotibial Joint Cartilage Thickness in Patients With Osteoarthritis". JAMA. 2019; Oct 8;322(14)

Disclosure of Interests: Hans Gühring Employee of: Merck KGaA, Anne-Christine Bay-Jensen Shareholder of: Nordic Bioscience A/S, Employee of: Full time employee at Nordic Bioscience A/S., Flavie Moreau Employee of: Merck KGaA, Jeppe Ragnar Andersen Shareholder of: Nordic Bioscience A/S., Employee of: Full time employee of Nordic Bioscience., Asger Reinstrup Bihlet Shareholder of: Nordic Bioscience A/S., Morten Karsdal Shareholder of: Nordic Bioscience A/S., Employee of: Full time employee at Nordic Bioscience A/S.

DOI: 10.1136/annrheumdis-2020-eular.3877

\section{FRI0405 CARTILAGE BIOMARKERS S-COLL2-1 AND S-COLL2-1NO2 ARE HELPFUL IN IDENTIFYING KNEE OSTEOARTHRITIS PATIENTS AT RISK OF DISEASE WORSENING}

Y. Henrotin $^{1}$, B. Costes ${ }^{2}$, M. Malaise ${ }^{3}$, D. Loeuille ${ }^{4}$, T. Conrozier $^{5}$, Y. Maugars ${ }^{6}$, F. Pelousse ${ }^{7}$, J. M. Lemaire ${ }^{8}$, T. Helleputte ${ }^{9}$, C. Tits $^{9}$, E. Cobraiville ${ }^{2}$, S. Pirson ${ }^{2}$, L. Garcia ${ }^{2}$, A. Labasse ${ }^{2}$, A. C. Hick ${ }^{2} .{ }^{1}$ University of Liège, Bone and Cartilage Research Unit, Liège, Belgium; ${ }^{2}$ Artialis SA, Liège, Belgium; ${ }^{3} \mathrm{CHU}$ Sart-Tilman, Liège, Belgium; ${ }^{4} \mathrm{CHU}$ Nancy, Nancy, France; ${ }^{5}$ Hopital Nord Franche-Comté, Belfort, France; ${ }^{6} \mathrm{CHU}$ Nantes, Nantes, France; ${ }^{7}$ Sodiray, Liège, France; ${ }^{8}$ Sodiray, Liège, Belgium; ${ }^{9}$ DNAlytics, Ottignies-Louvain-laNeuve, Belgium

Background: Coll2-1 is a peptide of 9 amino acid located in the triple helix of type II collagen molecule reflecting cartilage degradation (1). Coll2-1NO2 is the nitrated form of Coll2-1 and considered as a biomarker of the inflammatory-related cartilage degradation (2). This peptide is involved in osteoarthritis physiopathology since it was demonstrated that Coll2-1 induced synovitis in rat.

Objectives: To identify if biochemical markers s-Coll2-1 and s-Coll2-1NO2 are associated to knee osteoarthritis $(\mathrm{OA})$, focusing on pain, function as well as structural features assessed by MRI in various knee compartments and to assess their ability at predicting knee OA worsening.

Methods: 116 subjects with knee OA were followed during one year with pain, function and MRI evaluation (PRODIGE study, NCT02070224). Type II collagen-specific biomarker Coll2-1 and its nitrated form Coll2-1NO2 were directly measured in serum using immunoassays at baseline and after three, six and twelve months follow-up.

Results: sColl2-1 and sColl2-1NO2 were associated to several baseline knee features quantified with Whole-Organ Magnetic Resonance Imaging Score (WORMS). S-Coll2-1 was significantly correlated with bursitis $(r=0.29, P<0.01)$, bone attrition $(r=0.25, P=0.01)$, cysts $(r=0.24, P=0.02)$ and cartilage $(r=0.23$, $\mathrm{P}=0.03$ ) WORMS sub-scores for the whole joint as well as with the medial femorotibial joint sum score $(r=0.26, P=0.01)$ and medial femorotibial joint cartilage $(r=0.23, P=0.02)$. $s-C o l l 2-1 \mathrm{NO} 2$ was correlated with WORMS total score $(r=0.23, P=0.02)$, WORMS scores in the patellofemoral $(r=0.23, P=0.02)$ and medial femorotibial compartments $(r=0.21, P=0.03)$ and with osteophytes scores $(r=0.27, P<0.01)$. Baseline $s-C o l l 2-1 \mathrm{NO} 2$ was higher in subjects with a pain worsening (426.4 pg/mL [278.04-566.95]) as compared to non-progressors (306.84 [200.37-427.84]) over one year ( $A \cup C=0.655, P=0.015$ ).

Conclusion: Cartilage biomarkers s-Coll2-1 and s-Coll2-1NO2 are associated to several knee OA features quantified with WORMS scoring system on MRI. Serum values of Coll2-1NO2 are also associated to a worsening of target knee pain over one year. Coll2-1 and Coll2-1NO2, in association with other structural features, pain and function, could help at identifying OA phenotypes and patients at risk of OA worsening.

References:

[1] Mobasheri A, Lambert C, Henrotin Y. Coll2-1 and Coll2-1NO2 as exemplars of collagen extracellular matrix turnover - biomarkers to facilitate the treatment of osteoarthritis? Expert Rev Mol Diagn. 2019 Sep;19(9):803-812. doi: 10.1080/14737159.2019.1646641. Epub 2019 Sep 4.
[2] Lambert C, Borderie D, Dubuc JE, Rannou F, Henrotin Y. Type II collagen peptide Coll2-1 is an actor of synovitis. Osteoarthritis Cartilage. 2019 Nov;27(11):1680-1691. doi: 10.1016/j.joca.2019.07.009. Epub 2019 Jul 17.

Acknowledgments: PRODIGE study (NCT02070224) was performed in the framework of a convention between the Walloon region and ARTIALIS SA. (convention $n^{\circ} 6905$ ).

Disclosure of Interests: Yves Henrotin Grant/research support from: HEEL, TILMAN, Berenice Costes Employee of: Artialis SA, Michel Malaise: None declared, Damien Loeuille: None declared, Thierry Conrozier Consultant of: LABRHA, SANOFI, MEDAC, Yves Maugars: None declared, Franz Pelousse Shareholder of: Sodiray, Jean-Marc Lemaire Shareholder of: Sodiray, Thibault Helleputte Shareholder of: DNAlytics, Cedric Tits Employee of: DNAlytics, Elisabeth Cobraiville Employee of: Artialis SA, Sebastien Pirson Employee of: Artialis, Laetitia Garcia Employee of: Artialis, Alain Labasse Employee of: Artialis SA, Anne-Christine Hick Employee of: Artialis SA DOI: 10.1136/annrheumdis-2020-eular.3604

\section{FRI0406 \\ CARTILAGE REPAIR ACTIVITY DURING JOINT- PRESERVING TREATMENT MAY BE ACCOMPANIED BY OSTEOPHYTE FORMATION}

M. Jansen ${ }^{1}$, S. Mastbergen ${ }^{1}$, F. Watt ${ }^{2}$, E. Willemse ${ }^{1}$, T. Vincent ${ }^{2}$, S. Spruijt ${ }^{3}$, P. Emans ${ }^{4}$, R. Custers ${ }^{1}$, R. Van Heerwaarden ${ }^{5}$, F. Lafeber ${ }^{1} .{ }^{1}$ University Medical Center Utrecht, Utrecht, Netherlands; ${ }^{2}$ University of Oxford, Oxford, United Kingdom; ${ }^{3}$ HagaZiekenhuis, Den Haag, Netherlands; ${ }^{4}$ Maastricht University Medical Center, Maastricht, Netherlands; ${ }^{5}$ Kliniek ViaSana, Mill, Netherlands

Background: Knee joint distraction (KJD) is a joint-preserving treatment to postpone total knee arthroplasty (TKA) and has shown cartilage repair and clinical improvement in patients with severe knee osteoarthritis (OA), as has high tibial osteotomy (HTO). The observed cartilage repair activity could be related to an increase in transforming growth factor- $\beta 1$ (TGF $\beta-1$ ), which increases in the synovial fluid (SF) during KJD treatment. ${ }^{1}$ However, animal and ex vivo human studies have shown that TGF $\beta-1$ also induce formation of osteophytes, generally seen as an OA severity hallmark. Similarly, interleukin-6 (IL-6) was observed to increase in SF during KJD treatment and may also be associated with osteophytosis.

As such, we hypothesized that joint-preserving regenerative treatments demonstrating cartilage repair activity lead to general tissue (re)generation, including osteophytosis.

Objectives: To analyze osteophytosis after KJD and compare this to HTO and natural progression in knee $O A$.

Methods: $63 \mathrm{KJD}$ patients were included in several clinical trials, one of which was a randomized controlled trial comparing patients indicated for HTO, but treated with $\mathrm{KJD}\left(\mathrm{KJD}_{\mathrm{HTO}} ; \mathrm{n}=23\right)$ vs. patients treated with $\mathrm{HTO}(\mathrm{n}=46)$. All patients received standardized radiographs before and one and two years after treatment, used to measure osteophyte size. Only patients with measurements at baseline and two-year follow-up were included. As a control group for natural progression, untreated knee OA patients from Cohort Hip \& Cohort Knee (CHECK; $n=1002)$ were studied. Only patients who received a TKA during follow-up were included, using their last two measurements before treatment to reflect natural two-year progression ( $n=44)$.

A separate group of 20 patients treated with KJD in regular care underwent SF aspirations before and after treatment, and TGF $\beta-1$ and IL- 6 levels were measured by immunoassay (Mesoscale Discovery). Unstandardized radiographs were acquired before and one year after treatment, used to score osteophytes with the revised Altman score, resulting in a 0 (normal) to 12 (severe) whole-joint score. Only patients with radiographs and SF aspirations at both baseline and one-year follow-up were included.

Results: After two years, both KJD $(n=58)$ and HTO $(n=38)$ patients showed a significant increase in osteophyte size $(+6.2 \mathrm{~mm} 2$ and $+7.0 \mathrm{~mm} 2$ resp.; both $p<0.003$; figure 1), with no significant differences between the treatments ( $p>0.38$ ). Untreated CHECK patients who underwent TKA did not show significant two-year osteophyte changes before treatment $(+2.1 \mathrm{~mm} 2 ; p=0.207$; figure 1) and showed significant differences compared with KJD and HTO groups (both $\mathrm{p}<0.044)$. In the KJD SF aspiration group $(n=17)$, the Altman osteophyte score was not different at one year compared to baseline $(+0.2$ points; $p=0.653)$ and there was no association between baseline biomarker values and the baseline Altman osteophyte score, or between changes in these parameters (all $p \geq 0.28$ ). Trichotomization of patients in groups with a decrease, no change or increase in total Altman osteophyte score indicated that there was a statistically significant difference between the three groups in changes in TGF $\beta-1(p=0.044$ figure $2 A$ ), but not IL-6 ( $p=0.898$; figure $2 B$ ).

Conclusion: After KJD treatment, joint space widening and clinical improvement are accompanied by osteophytosis. Similar results were observed after treatment with HTO, suggesting effects occur in regenerative joint-preserving 